\title{
Review Article \\ The Role of Oxidative Stress in Early Brain Injury after Subarachnoid Hemorrhage
}

\author{
M. Jelinek $\mathbb{D}^{1,}{ }^{1,2}$ M. Jurajda, ${ }^{1}$ and K. Duris $\mathbb{D}^{1,3}$ \\ ${ }^{1}$ Department of Pathophysiology, Faculty of Medicine, Masaryk University, Brno, Czech Republic \\ ${ }^{2}$ Department of Experimental Biology, Faculty of Science, Masaryk University, Brno, Czech Republic \\ ${ }^{3}$ Department of Neurosurgery, The University Hospital Brno and Faculty of Medicine, Masaryk University, Brno, Czech Republic
}

Correspondence should be addressed to K. Duris; kduris@med.muni.cz

Received 21 September 2020; Revised 16 October 2020; Accepted 31 October 2020; Published 19 November 2020

Academic Editor: Robert Ostrowski

Copyright (c) 2020 M. Jelinek et al. This is an open access article distributed under the Creative Commons Attribution License, which permits unrestricted use, distribution, and reproduction in any medium, provided the original work is properly cited.

This review focuses on the problem of oxidative stress in early brain injury (EBI) after spontaneous subarachnoid hemorrhage (SAH). EBI involves complex pathophysiological mechanisms, including oxidative stress. In the first section, we describe the main sources of free radicals in EBI. There are several sources of excessive generation of free radicals from mitochondrial free radicals' generation and endoplasmic reticulum stress, to hemoglobin and enzymatic free radicals' generation. The second part focuses on the disruption of antioxidant mechanisms in EBI. The third section describes some newly found molecular mechanisms and pathway involved in oxidative stress after EBI. The last section is dedicated to the pathophysiological mechanisms through which free radicals mediate early brain injury.

\section{Introduction}

Spontaneous subarachnoid hemorrhage $(\mathrm{SAH})$ is a serious form of hemorrhagic stroke with high morbidity and mortality. SAH patients suffer from an initial mortality of $15-20 \%$, $40 \%$ die within one month, and around one-third of survivors have serious morbidity in the form of neurological deficits [1]. The majority of SAH cases are caused by a nontraumatic rupture of a cerebral aneurysm. For decades, research concerning $\mathrm{SAH}$ has focused on delayed cerebral ischemia (DCI) and delayed cerebral vasospasms (CVS). However, controversial results of the studies with clazosen$\tan$ (the CONSCIOUS studies) and lack of prevention against DCI are why the trend in SAH research has been shifting from DCI and delayed CVS to early brain injury [2].

Early brain injury (EBI) encompasses complex pathophysiological changes in the central nervous system (CNS) in the first 72 hours after the onset of bleeding and before the development of CVS. It has been suggested that EBI mechanisms strongly determine the morbidity and mortality of SAH patients and is important in the development of impairments like CVS, DCI, and associated delayed cerebral ischemic deficit (DIND) [2]. The range of pathophysiological impairments that get triggered during EBI includes ionic, biochemical, molecular, and vascular changes, oxidative stress, inflammation, blood-brain barrier (BBB) dysfunction, apoptosis, and necrosis [2].

Oxidative stress is strongly associated with pathophysiological mechanisms of EBI after SAH [3]. Cellular respiration impairment results in production of free radicals, which are molecules with an unpaired valence electron and are highly chemically reactive. Disruption of metabolism of neurons due to hypoxia and the resultant changes in cellular oxidative state are associated with oxidative stress [4]. Therefore, the brain, with its high metabolic demands, is especially sensitive to free radicals' injury whenever there is a disruption in the cellular respiration. In EBI, an imbalance is struck between production of reactive oxygen species (ROS) and intrinsic antioxidant system's ability to counteract or repair the damage. This imbalance favors ROS and leads to damage to the 


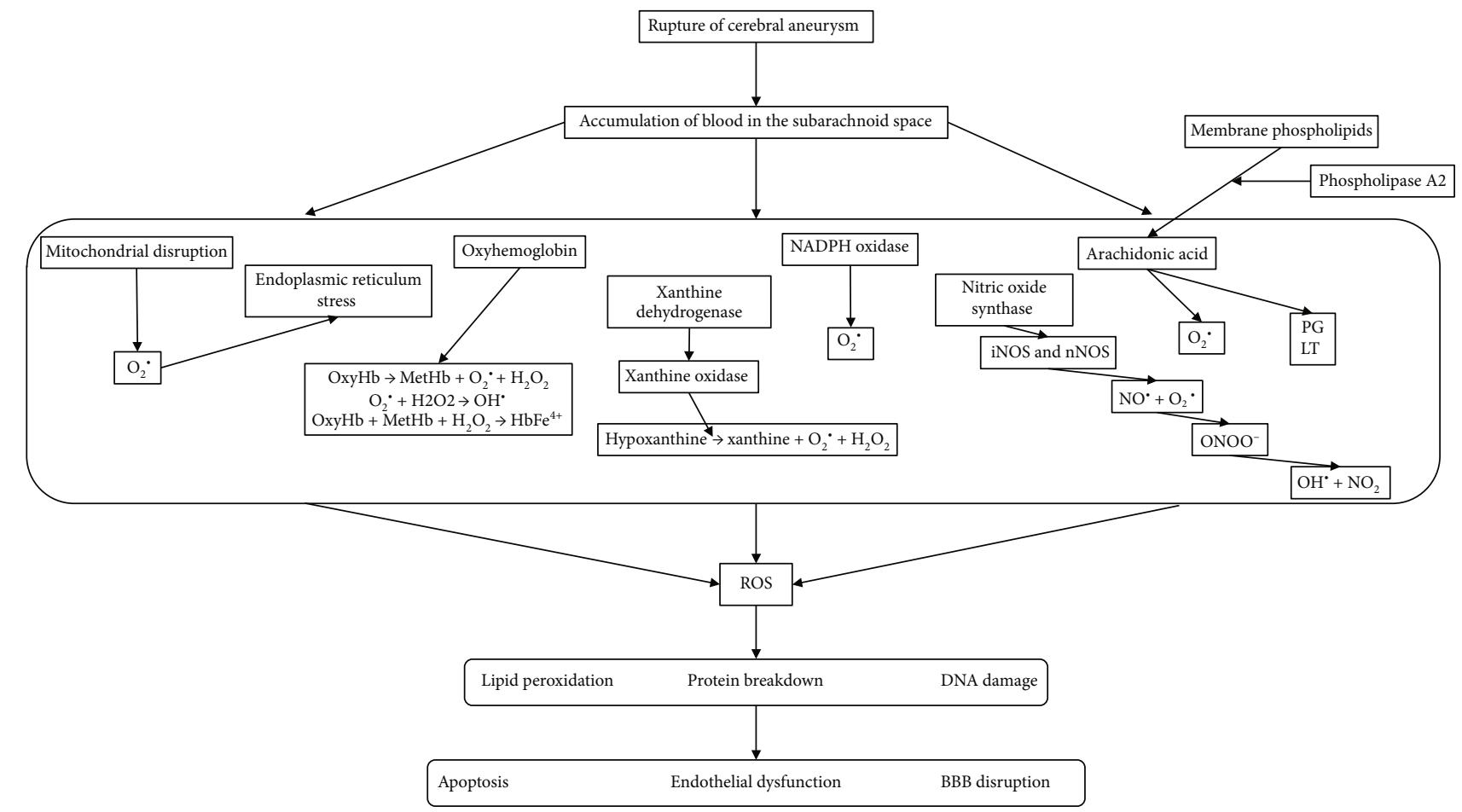

FIGURE 1: Diagram of major sources of oxidative stress in the pathophysiology of EBI after SAH. BBB: blood-brain barrier; iNOS: inducible nitric oxide synthase; LT: leukotriene; MetHb: methemoglobin; nNOS: neuronal nitric oxide synthase; OxyHb: oxyhemoglbin; PG: prostaglandin; ROS: reactive oxygen species.

brain. The most common free radicals involved in oxidative stress after SAH are superoxide anion, hydroxyl radical, nitric oxide, and peroxynitrite [5].

This review discusses known mechanisms of excessive free radicals' production, a disruption of antioxidant mechanisms, basic molecular signaling pathways, and brain injury associated with oxidative stress in EBI after SAH (Figure 1).

\section{Free Radicals Mediated Early Brain Injury}

ROS are key mediators of pathophysiological mechanisms involved in EBI after SAH. There is an early generation of ROS in the form of superoxide anion, hydroxyl radical, hydrogen peroxide, NO, and peroxynitrate after SAH [6]. There are also several antioxidant systems that try to deal with ROS. However, their levels are inadequate to deal with such an excess of ROS formation, leading to an imbalance between ROS production and antioxidant systems in the brain after SAH.

There are several mechanisms of ROS-associated injury after SAH. ROS damage the vascular smooth muscle and endothelium [2]. This involves damage to membrane-bound phospholipids, which leads to production of second messengers like inositol-3-phosphate (IP3) and diacylglycerol (DAG) and derivates of arachidonic acid such as leutrotriene C4 and prostaglandin D2 [5]. Their presence leads to vasoconstriction and subsequently to delayed cerebral vasospasms [2, 5]. These pathophysiological changes lead to the disruption the blood-brain barrier (BBB) and consequently to cerebral edema [2]. ROS induce enzymes of apoptotic pathways such as p53 and caspase-3 and caspase-9 leading to cell death [7].

Oxidative stress in EBI could be linked to cerebral vasospasms and delayed cerebral ischemia (DCI). The changed levels of vasodilators (NO) and vasoconstrictors (endothelin 1) or tissue unresponsiveness can lead to microvascular dysfunction and to cerebral vasospasms $[8,9]$. Furthermore, microvascular dysfunction can lead to parenchymal hypoperfusion [8]. Cerebral vasospasms and parenchymal hypoperfusion can be linked to cell death (apoptosis, necrosis, and autophagy) in neurons [8]. Other pathophysiological mechanisms like microthrombosis and neuroinflammation, disruption of the $\mathrm{BBB}$, and the subsequent cerebral edema also contribute to the neuronal cell death $[2,8]$. Neuronal cell death along with other complications (like hydrocephalus and systemic inflammatory response syndrome) can lead to DCI.

\section{Main Sources of Free Radicals in EBI after SAH}

There are several sources that contribute to excessive free radicals' production in EBI after SAH. The two main sites that can tip the scales in free radicals' favor are mitochondrial overproduction, hemoglobin autooxidation, and inflammation. Additionally, endoplasmic reticulum stress can also contribute to high free radicals' production and consequently oxidative stress in EBI. Finally, there are also several enzymatic pathways that can generate free radicals. 
3.1. Mitochondrial Free Radicals' Generation. The main source of free radicals in EBI is the leakage of superoxide anions from mitochondria. It is caused by the ischemic disruption of the electron transfer chain [3]. In normal (physiological) conditions, electrons leak from the transport chain and react with oxygen- $\left(\mathrm{O}_{2^{-}}\right)$producing superoxide anion $\left(\mathrm{O}_{2}{ }^{-}\right)$. Superoxide is then cleared by superoxide dismutase, which converts superoxide radicals to molecular oxygen and hydrogen peroxide [10]. However, after a period of ischemia that occurs in EBI pathophysiology, the mitochondria produce excessive amounts of free radicals that can no longer be cleared by antioxidants (such as superoxide dismutase) [2]. The overload of free radicals that cannot be cleared by antioxidants then have the potential to cause damage to lipids, proteins, and DNA [3]. In general, the production of ROS is highest when components of the electron transfer chain are maximally reduced [11].

Under physiological conditions, mitochondria display a high electrochemical gradient which is generated by the proton pumping components of the respiratory electron transport chain. This gradient is the driving force for phosphorylation of adenosine diphosphate (ADP) to adenosine triphosphate (ATP) and closing off $\mathrm{Ca}^{2+}$ from the cytosol. However, EBI after $\mathrm{SAH}$ and the subsequent ischemia cause an exaggerated $\mathrm{Ca}^{2+}$ accumulation in the mitochondria [12]. This accumulation leads to disruption of the mitochondrial membrane potential and opening of membrane permeability transition pores (MPTPs) [13]. MPTPs are multiprotein channel complexes that span the inner and outer mitochondrial membranes, allowing solutes of less than 1500 Daltons ( $\mathrm{Da}$ ) to cross the membrane from the matrix to the cytosol $[12,13]$. Mitochondria tries to reestablish an electrochemical gradient by increasing substrate oxidation and $\mathrm{O}_{2}$ consumption, which leads to more free electrons interaction with $\mathrm{O}_{2}$ and the creation of superoxide [3]. To support this, state 4 mitochondrial respiration and decreased respiratory control ratios that are associated with increased ROS production has been recorded after SAH [14]. Discussion continues about the possible pros and cons of targeting the MPTPs as a therapeutic strategy after SAH [12]. Specific molecules had been used for the inhibition of MPTPs, such as immunosuppressant cyclosporin A and NIM811, reducing oxidative damage [12].

Mitochondrial dysfunction is also thought to contribute to oxidative stress and the pathophysiological mechanisms of EBI. Mitochondrial dynamics, such as mitochondrial fission and fusion imbalance, have been recently recognized as some of the key components of mitochondrial dysfunction [15]. Their mechanisms in attenuating oxidative stress and apoptosis in EBI after SAH have been recently explored by Wu et al. [15] and Zhang et al. [16].

The protein Drp1 plays an important role in the mitochondrial dysfunction [15]. Mdivi-1 is a selective inhibitor of Drp1, a mitochondrial fission protein [15]. It has the ability to penetrate the $\mathrm{BBB}$, giving it importance in CNS diseases [15]. Mdivi-1 treatment alleviated oxidative stress during EBI elevation of superoxide dismutase (SOD) activity and reduced markers of lipid, protein, and DNA oxidative injury malondialdehyde (MDA), 3-nitrotyrosine (3-NT), and 8hydroxy-2' -deoxyguanosine (8-OHdG) [15].

Zhang et al. [16] researched docosahexaenoic acid (DHA) effects on EBI after SAH. DHA is an omega-3 fatty acid. In in vitro study using $\mathrm{OxyHb}$ to simulate the effects of hemorrhagic stroke [16-18], DHA attenuated mitochondrial disorders (fission and fusion imbalance), which possibly contributes to its antioxidative and antiapoptotic effects [16]. The mechanism of DHA neuroprotective properties has to do with decreased mitochondrial fragmentation in after SAH as well as the expression of Drp1 [16]. Furthermore, DHA was able to improve neurological deficits and attenuate brain edema, as well as significantly limit the increase of SAH-induced oxidative stress and apoptosis [16]. Taken together, oxidative stress induced by an imbalance of mitochondrial dynamic was improved by DHA in both in vitro and in vivo conditions [16]. Suggesting that improved mitochondrial dynamics may be responsible for the antioxidative effects of DHA.

3.2. Endoplasmic Reticulum Stress. Another important mechanism that is linked with oxidative stress is an endoplasmic reticulum (ER) stress. Their interaction has been found in acute central nervous system injuries, such as EBI after SAH [19]. EBI causes ROS overproduction, leading to the disorder of ER redox and cell apoptosis [19]. ER stress leads to ROS overproduction making it a vicious cycle and further worsening the oxidative damage [20]. Combination of therapies that would alleviate both stresses could be of potential therapeutic interest. Resveratrol has been shown to reduce both oxidative and ER stresses [21]. ER stress was also shown to be alleviated by apelin-13 through inhibition of activating transcription factor 6 (ATP6) in EBI after SAH [22], thus, alleviating ER stress-mediated neuronal apoptosis [22]. Similarly, apelin-13 has been shown to alleviate ER stress via the apelin receptor (APJ) through the AMPK/TXNIP/NLRP3 signaling pathway, which downstream signaling results in inflammation and cellular apoptosis [23-25].

3.3. Hemoglobin Free Radicals' Generation. Another major source of free radicals and oxidative stress in the pathophysiology of EBI after SAH is autooxidation of hemoglobin. The introduction of oxyhemoglobin (oxyHb) into the subarachnoid space and consequently to the cerebrospinal fluid (CSF) is a major producer of superoxide anion $\left(\mathrm{O}_{2}{ }^{-}\right)$and hydrogen peroxide $\left(\mathrm{H}_{2} \mathrm{O}_{2}\right)$ as it goes through autooxidation to methemoglobin [3]. The iron ion liberated from oxyHb propels the generation of hydroxyl radical $\left(\mathrm{OH}^{-}\right)$from $\mathrm{O}_{2}{ }^{-}$ and $\mathrm{H}_{2} \mathrm{O}_{2}$ (both from metHb) [26]. Interaction between metHb and oxyHb with $\mathrm{H}_{2} \mathrm{O}_{2}$ also generates ferryl hemoglobins $\left(\mathrm{HbFe}^{4+}\right)$, which are strong oxidizing agents [26].

Many studies mention the oxidizing effects of hemoglobin on lipids, proteins, and DNA $[3,26,27]$. Products of hemolysis in the subarachnoid space also increase cytochrome c-mediated DNA fragmentation [27]. A study by [26] found an increased nonheme iron and iron-handling proteins during EBI. They also introduced iron chelator DFX into the system that helped reduce SAH-induced cell damage and changes related to hemoglobin-related oxidative 
stress, making it a possible therapeutic target for patients with SAH [26].

3.4. Enzymatic Free Radicals' Generation. Besides free radicals generated by mitochondria and hemoglobin autooxidation, there are several other enzymatic pathways that can produce free radicals. The intracellular accumulation $\mathrm{Ca}^{2+}$ in neurons (due to extracellular hemoglobin and ischemia) can produce free radicals through activation of several prooxidative mechanisms like phospholipases, nitric oxide synthase, NADPH oxidase, and xanthine oxidoreductase.

3.4.1. Arachidonic Acid Derivates. Arachidonic acid (AA) is released from phospholipids in the cytoplasmic membrane by phospholipase A2 (PLA2). AA is then processed by cyclooxygenase, lipoxygenase, or cytochrome P450, where each of these pathways can produce superoxide anion as their byproduct [28]. These mechanisms are considered to be an important source of free radicals in brain injury and ischemia [28]. Increased PLA2 activity, as well as cyclooxygenase and lipoxygenase, has been found to follow $\mathrm{SAH}[3,29-31]$. Inhibition of these pathways had a positive clinical impact (on vasospasms), but it is unclear if it was through reduction of oxidative stress. A study by [32] focused on inhibition of leukotriene B4 (LTB4). LTB4 is a highly potent chemoattractant for leukocytes (mainly neutrophils). LTB4 is generated from arachidonic acid (AA) by cytosolic phospholipase A2, 5-lipoxygenase, and leukotriene A4 hydrolase [33]. Activated neutrophils are a source of oxidative stress because of their respiratory burst and are drawn to the subarachnoid space by, among others, LTB4 [34]. Reduced LTB4-driven neutrophil infiltration decreases ROS and MDA and increases levels of SOD and glutathione peroxidase (GSH-Px) during EBI [32]. Therefore, inhibition of LTB4 synthesis may decrease the neutrophil-generated oxidative stress. Furthermore, blockage to LTB4 synthesis reduces translocation of nuclear factor kappa B (NF- $\kappa \mathrm{B})$ and its DNA-binding activity [32]. It leads to the alleviation of the NF- $\kappa \mathrm{B}$-mediated inflammation during EBI after SAH [32]. Furthermore, the reduction of synthesis of LTB4 could decrease neutrophil recruitment to the brain parenchyma after SAH. The specific mechanism of LTB4/NF- $\kappa$ B-mediated inflammation remains unknown.

3.4.2. Nitric Oxide in Early Brain Injury. Generally, there are two main systems that play crucial role in oxidative stress after EBI. One of them is the oxygen-based free radicals and the other is nitrogen-based free radicals. Nitric oxide has a versatile function in the CNS. It is an important regulator of the cerebral blood flow (CBF); it inhibits platelet adherence, reduces adherence of leukocytes, suppresses vessel injury, acts as a neurotransmitter and finally a free radical [9].

Nitric oxide $(\mathrm{NO})$ as a free radical $\left(\mathrm{NO}^{\bullet}\right)$ is generated by nitric oxide synthase (NOS). NOS has three isoforms: endothelial (eNOS), inducible (iNOS), and neuronal (nNOS) [35]. Two main two types involved in EBI are iNOS and nNOS [35]. NO levels in the first 24 hours after SAH decrease, recover, and increase, making it a very dynamic process [9]. Cerebral ischemia increases NOS activity and gene expression [36]. In EBI, around hour 6 after the initial bleeding, the major producer of $\mathrm{NO}^{\circ}$ is iNOS [9].

There are three main phases of the $\mathrm{NO}$ activity that are described in EBI. Information about phase I and II come from experimental animal studies. Phase III has been studied in both animals and humans [37]. Phase I describes the first hours after SAH and is characterized by an acute decrease in cerebral NO level [38]. Cerebral vessels are constricted and $\mathrm{CBF}$ is reduced [39]. NO-dependent vasodilation is impaired in this early stage of EBI after SAH [39]. However, NO donor increases the reduced $\mathrm{CBF}$ at this stage [39]. Interestingly, in occlusive ischemic stroke, there is an increase in cerebral NO in phase I. Phase II defines hours 1-6 in which NO levels recover to their baseline [9]. The CBF remains reduced, which suggests that NO produced during phase II is unable to recover the initial CBF [40]. Also, NO donor has little effect on CBF [9]. Phase III encompasses the rest of the EBI period (hour 6-72) [9]. Arterial response to NO is reduced at this stage, possibly due to alterations in cGMP pathways [41]. Increase in NO levels in phase III has been linked to oxidative damage in the brain [9].

$\mathrm{NO}$ can interact with $\mathrm{O}_{2}{ }^{-}$to form peroxynitrite $\left(\mathrm{ONOO}^{-}\right)$, which can then turn into $\mathrm{OH}^{-}[42] . \mathrm{O}_{2}{ }^{-}, \mathrm{ONOO}^{-}$, and $\mathrm{OH}^{-}$are neurotoxic free radicals, damaging the cell and leading to cell death [42]. The presumed mechanisms of NO oxidative damage involve lipid peroxidation of the cell membranes and DNA and mitochondrial damage, leading to apoptosis [9].

On the other hand, NO could have beneficial effects on the cerebral blood flow $[39,43]$. In addition, it has been hypothesized that $\mathrm{NO}^{\circ}$ may also possess neuroprotective properties due to its ability to modify the NMDA receptor response and reduce toxicity [43].

3.4.3. NADPH Oxidase. NADPH oxidase (NOX) is a membrane-bound enzyme complex found on the extracellular side of the cytoplasmic membrane and may produce $\mathrm{O}_{2}^{-}$[44]. NOX has been found to contribute to oxidative stress in in vitro studies [45] and increased expression of NOX in SAH could be associated with elevated oxidative stress [46]. Reduction of NOX-associated free radicals' generation was associated with reductions in mortality, neurological deficits, and neuronal cell death [46]. Direct inhibition of NOX in EBI after SAH has not yet been studied in SAH.

3.4.4. Xanthine Dehydrogenase. Xanthine dehydrogenase $(\mathrm{XDH})$ is an enzyme homodimer found among others in the endothelium [47]. It is involved in the oxidative metabolism of purines creating uric acid [47]. XDH does not produce free radicals directly, rather it is converted to xanthine oxidase (XO) during ischemia, hypoxia, and excitotoxicity [47]. XO then catalyzes the oxidation of hypoxanthine to xanthine creating uric acid, $\mathrm{O}_{2}{ }^{-}$and $\mathrm{H}_{2} \mathrm{O}_{2}$ [3]. The general impact of $\mathrm{XO}$ inhibition during SAH is being discussed because of other more significant pathways that generate free radicals.

\section{Disruption of Antioxidant Mechanisms}

There are several protective systems that try to balance out the production of free radicals. Superoxide dismutase 
(SOD), glutathione peroxidase (GSH-Px), and catalase (CAT) are important enzymatic scavengers during normal cellular respiration [3]. However, in EBI after SAH, these systems become downregulated, overwhelmed, or modulated leading to reduction of their antioxidative capacities [14].

Under physiological conditions, SOD produces $\mathrm{H}_{2} \mathrm{O}_{2}$ from $\mathrm{O}_{2}^{-} ; \mathrm{H}_{2} \mathrm{O}_{2}$ is then scavenged by GSH-Px to prevent formation of the more dangerous $\mathrm{OH}^{-}$[48]. However, in $\mathrm{SAH}$, there is an increased activity of $\mathrm{Cu}-\mathrm{SOD}$ and $\mathrm{Zn}-\mathrm{SOD}$ and an increase in the SOD/GSH-Px ratio [14]. This leads to excess production of $\mathrm{OH}^{-}$which cannot be scavenged by GSH-Px, meaning increased SOD/GSH-Px ratio has the potential to be a significant source of free radicals.

Several molecules have been studied due to their antioxidant capacity for alleviating oxidative stress in EBI after SAH $[16,21,49-52]$. Resveratrol was studied as one such molecule by Xie et al. [21]. Resveratrol is a natural phenol with broad bioactive capacity. Resveratrol treatment decreased the excessive production of ROS and MDA, which are indicators of oxidative stress [21]. Resveratrol has been reported to inhibit protein carbonylation and nitration and lipid peroxidation, attenuating oxidative stress [21]. Furthermore, resveratrol has an antioxidant capacity of activating endogenous mechanisms, such as upregulation of Nrf2 and HO-1 in EBI after SAH [21]. Under pathological conditions, such as oxidative stress during EBI, the Nrf2 pathway gets activated and triggers the antioxidant enzyme expression, including $\mathrm{HO}-1$, attenuating cellular oxidative stress [53, 54]. HO-1 exerts its antioxidant properties by catalyzing the degradation of heme [55]. Therefore, induction of the Nrf2 and HO- 1 systems result in protective mechanisms against cellular oxidative stress. Another molecule, cysteamine, was studied by Zhang et al. [51]. Cysteamine is a product of coenzyme A degradation. Intraperitoneal administration of cysteamine was shown to have positive antioxidative and antiapoptotic effects in EBI after SAH [56]. Similar to other molecules discussed in this review, cysteamine reduced ROS, MDA, 3-NT, and 8-OHdG levels and increased GSHPx activity [56]. The mechanism of effect of cysteamine is not yet known.

\section{Molecular Signaling}

Molecular signaling, different intracellular pathways, their interactions, and effects are important for our understanding not only of the mechanisms of oxidative stress after EBI but also generally the pathophysiology of SAH. Although our knowledge in this field is still limited, there are some known mechanisms that may help understand the underlying mechanisms of what is happening inside the cell because of oxidative stress during EBI.

An important signaling pathway that gets activated in EBI after SAH is proapoptotic (and proinflammatory) mitogen-activated protein kinase (MAPK) [51, 57]. Oxidative stress is a strong inducer of MAPK signaling [50]. Antioxidants have been shown to attenuate the MAPK pathway signaling [58]. Antioxidant naringin was found to restore the decreasing levels of SOD, CAT, and GSH-Px [59]. Naringin has also reduced levels of phosphorylated ERK1/2, JNK, and p38, which are downstream molecules of MAPK signaling [59]. This indicated that naringin could have potential antiapoptotic and antioxidative effects due to its targeting of MAPK signaling.

In addition, Dou et al. [60] have studied the involvement of tumor necrosis factor receptor-associated factor 6 (TRAF6) on oxidative stress. TRAF6 is a protein with ubiquitin ligase activity that mediates signal transduction from members of the TNF receptor superfamily [60]. Toll-like receptor 4 (TLR4) has also been suggested as an upstream molecule (receptor) of the TRAF6 signaling pathway [60]. TRAF6 plays a possible role as an intersection between activating $\mathrm{NF}-\kappa \mathrm{B}$ pathway, which is an important regulator in immune development, immune response, inflammation, and cancer, and MAPK/ERK pathway, which controls adaptive responses to intracellular and extracellular stresses [6063]. The final downstream molecules NF- $\kappa$ B and AP-1 then can add to a great number of pathophysiological processes. TRAF6 levels increase during EBI (peaking at 24 hours) [60]. The connection between TRAF6 and oxidative stress in EBI after SAH may lie in its capacity to mediate the production of ROS and ability to translocate to mitochondria and ubiquitinate ECSIT, which is important for assembly and stabilization of respiratory complex I, and consequently to further increasing production of ROS in mitochondria [60]. Thus, administration of TRAF6 inhibitors could be of a potential interest in the reduction of oxidative stress in EBI. Liu et al. [64] has shown the TRAF6 inhibitor (sophoridine) potential in reducing the size of infarctions in ischemic injury.

In addition, another pathway, possibly involved in signaling due to oxidative stress in EBI, is the Mas/PKA/CREB/UCP-2 pathway [65]. Mo et al. [65] studied AVE 0991 which through this pathway reduced oxidative stress and neuronal apoptosis in EBI around hour 24 after the onset of bleeding. AVE 0991 (AVE) is a nonpeptide analog of angiotensin (1-7) [65]. It signals through receptor Mas, which is a G-protein coupled receptor and has been identified as a component of the renin-angiotensin system in the brain [65]. The suggested mechanism of reduced oxidative stress and neuronal apoptosis involves upregulation of PKA-C alpha, phosphorylated CREB, a transcription factor, UCP-2, which is a controller of mitochondria-derived reactive oxygen species, and $\mathrm{Bcl}-2$, apoptosis regulatory protein [65], as well as the downregulation of Bax, which is an apoptotic activator, and Romo-1, which can induce production of reactive oxygen species during EBI [65].

\section{Molecular Mechanisms of Free Radicals Mediated Early Brain Injury}

Oxidative stress or consequently free radicals lead to neuronal damage by promoting lipid peroxidation, protein breakdown, DNA damage which in turn contributes to apoptosis, endothelial dysfunction, and blood-brain barrier (BBB) disruption [3]. The effects of oxidative stress known as lipid peroxidation alter membrane fluidity and permeability [27]. Oxidation of proteins can change the functions of enzymes and receptors. 
Free radicals' damage can also initiate apoptotic cascades or shift them into necrosis [66, 67]. Apoptosis has been shown to be induced by oxidative stress, either by increasing p53, induction of cytochrome c release, and activation of caspase- 9 and caspase-3, or through p38 mitogen-activated protein kinase (MAPK) and signal-regulated kinase- (ERK) mediated apoptosis [66, 67]. Some studies [50,68] focus on molecules (apigenin, paeoniflorin) that could be of potential therapeutic interest because of their antioxidative and antiapoptotic effects. Apigenin (API) is a flavone naturally occurring in plants (it is mainly isolated from Hypericum perforatum) [50]. It has been found that API can reduce the concentration of ROS and elevate levels of GSH-Px and SOD during EBI after SAH [59], although the underlying mechanism has not been described. It was also reported that API could have antiapoptotic effects due to its ability to reverse the upregulation of Bax and caspase-3 [59]. Bax-dependent caspase- 3 has been reported as the initiation molecule of neuronal apoptosis [69]. Oxidative stress upregulates Bax and caspase-3 during EBI after SAH [59]. API also has antiinflammatory effects through the inhibition of the TLR4mediated inflammatory pathway [70]. Taken together, API is a molecule of great potential in EBI after SAH due to its antioxidative, anti-inflammatory, and antiapoptotic properties, nevertheless further research is still needed. Similar results as with API were reached with paeoniflorin. Paeoniflorin is a natural monoterpene glucoside from the root of Paeonia lactiflora [68]. Paeoniflorin was able to decrease levels of proinflammatory cytokines IL- $1 \beta$, IL- 6 , and TNF $\alpha$, which are upregulated during EBI [68]. Similar to API, the concentration of ROS has decreased and levels of GSH-Px and SOD have increased during EBI after SAH [68]. Antiapoptotic effects through Bax-dependent caspase- 3 were also observed [68].

Blood-brain barrier (BBB) disruption is an important part of the SAH pathophysiological mechanisms. Microvessels and their tight junctions help to constitute the BBB and have been shown to be disrupted in EBI, because their endothelial cells are susceptible to injury by oxidative stress [71]. This effectively leads to BBB dysfunction and subsequent cerebral edema. Antioxidants (edaravone and curcumin) have been shown to prevent MMP-9 expression and activation $[49,52]$. Subsequently blocking MMP-9 mediated degradation of claudin-5 and occludin, damaging tight junctions $[49,52]$. The specific regulatory signal of MMP-9 is still unknown, but possible candidates and interleukin- (IL-) $1 \beta$, IL-6, tumor necrosis factor- $\alpha(\mathrm{TNF} \alpha)$, and NF- $\kappa \mathrm{B}$ [49].

\section{Clinical Implications and Future Directions}

As mentioned above, there are many sources of free radicals' production in the EBI after SAH. There is also promising potential for using free radicals' scavengers, antioxidants, and reinforcing the innate antioxidative mechanisms that already exist in the brain. Many of these compounds, like DHA [16], resveratrol [21], API [50], paeoniflorin [68], and ebselen [72], have been described in this review. These antioxidants have been used in clinical and experimental trials with promising results.
Some clinical research has also been focused on the use of antioxidant micronutrients like vitamins $\mathrm{C}$ and $\mathrm{E}$, copper, selenium, and zinc [73]. The overall results of Berger et al.'s [73] study did not find significant all-around improvement of SAH patient's conditions, which is understandable due to the many pathophysiological mechanisms of SAH. However, the administration antioxidant micronutrients significantly blunted the associated inflammation [73].

The suggested therapeutic window is difficult to pinpoint because of the lack of a larger clinical research. However, based on the article by Bergen et al. [73] article, the micronutrient antioxidant intervention started at 24 hours after the initial bleeding. For the first 2 days, they used double dosage, and for the last 3 days, they used therapeutic dose as described in their article.

Other pharmacological research has focused on the use of lipid peroxidation inhibitor tirilazad and the superoxide scavenger PEG-SOD [74]. The therapeutic window for PEG-SOD is around 4 hours after the initial bleeding and tirilazad has the therapeutic window of 1 to 4 hours after the initial bleeding [74]. The results for both antioxidant drugs failed to produce the desired results [74]. However, tirilazad produced statistically significant increase in survival [74]. Therefore, further research with an LP-inhibiting antioxidant might be more relevant to the treatment of SAH.

Although the practical applicability of antioxidative treatment is still limited, the potential for therapeutic use exists and is worth investigating. Currently, there is no antioxidative treatment being routinely used in SAH patients. It can be contributed to the fact that oxidative stress is merely one parameter of a boarder EBI problematic. However, it is possible that targeted antioxidant therapy could be effective as one component of a larger treatment regime that would address the many components and mechanisms that are involved in EBI after SAH.

\section{Conclusion}

The content of this article could be summarized as a review of various aspects of oxidative stress associated with early brain injury (EBI) after subarachnoid hemorrhage (SAH). Mechanisms of oxidative stress after EBI are a subject of an extensive research. EBI and oxidative stress, as a part of its pathophysiology, are of great importance in understanding the complex mechanisms of SAH. Mechanisms that can lead to severe complications (like cerebral vasospasms (CVS), delayed cerebral ischemia (DCI), and delayed ischemic neurological deficit (DIND)) get activated within minutes after the onset of bleeding. That is why the focus of SAH research has been shifting towards EBI.

Under physiological conditions the brain is able to deal with the generation of ROS through different antioxidative mechanisms. However, when there is such an excessive production of free radicals like in $\mathrm{SAH}$, the antioxidative capacities are overused. It has serious negative effects and worsens the conditions and general outcome.

Oxidative stress comes from several sources in EBI after SAH. Mitochondrial disruption and subsequent endoplasmic reticulum stress play an important role in excessive production 
of ROS. Hemoglobin autoxidation and various interactions of $\mathrm{OxyHb}$ and $\mathrm{MetHb}$ also lead ROS overproduction. Unregulated enzymatic pathways like xanthine dehydrogenase, NADPH oxidase, NOS, and derivates of arachidonic acid processing can also be a major source of ROS.

The importance of studying oxidative stress after EBI lies in its multisource nature. Oxidative stress is among others involved in apoptosis, endothelial dysfunction, and BBB disruption. This is due to the ability of ROS to cause lipid peroxidation, protein breakdown, and DNA damage.

Oxidative stress after EBI poses as a potential therapeutic target and needs to be examined and understood in more detail. It would be helpful to integrate basic oxidative stress research of molecular signaling with $\mathrm{SAH}$-specific characteristics. Further research in this field could provide a better care for SAH patients.

\section{Conflicts of Interest}

The authors declare that they have no conflict of interest.

\section{Funding}

This work was supported by the projects MUNI/InGA/SUP/24/2020 and MUNI/A/1453/2019.

\section{References}

[1] J. Pang, J. Peng, P. Yang et al., "White matter injury in early brain injury after subarachnoid hemorrhage," Cell Transplantation, vol. 28, no. 1, pp. 26-35, 2018.

[2] F. A. Sehba, R. M. Pluta, and J. H. Zhang, "Metamorphosis of subarachnoid hemorrhage research: from delayed vasospasm to early brain injury," Molecular Neurobiology, vol. 43, no. 1, pp. 27-40, 2011.

[3] R. E. Ayer and J. H. Zhang, "Oxidative stress in subarachnoid haemorrhage: significance in acute brain injury and vasospasm," in Cerebral Vasospasm: New Strategies in Research and Treatment, T. Kırış and J. H. Zhang, Eds., vol. 104 of Acta Neurochirurgica Supplement, pp. 33-41, Springer Vienna, Vienna, 2008.

[4] M. E. Watts, R. Pocock, and C. Claudianos, "Brain energy and oxygen metabolism: emerging role in normal function and disease," Frontiers in Molecular Neuroscience, vol. 11, 2018.

[5] F. A. Sehba and J. B. Bederson, "Mechanisms of acute brain injury after subarachnoid hemorrhage," Neurological Research, vol. 28, no. 4, pp. 381-398, 2006.

[6] S. Chen, H. Feng, P. Sherchan et al., "Controversies and evolving new mechanisms in subarachnoid hemorrhage," Progress in Neurobiology, vol. 115, pp. 64-91, 2014.

[7] W. J. Cahill, J. H. Calvert, and J. H. Zhang, "Mechanisms of early brain injury after subarachnoid hemorrhage," Journal of Cerebral Blood Flow \& Metabolism, vol. 26, no. 11, pp. 13411353, 2006.

[8] J. R. Geraghty and F. D. Testai, "Delayed cerebral ischemia after subarachnoid hemorrhage: beyond vasospasm and towards a multifactorial pathophysiology," Current Atherosclerosis Reports, vol. 19, no. 12, 2017.

[9] F. A. Sehba and J. B. Bederson, "Nitric oxide in early brain injury after subarachnoid hemorrhage," in Early Brain Injury or Cerebral Vasospasm, H. Feng, Y. Mao, and J. H. Zhang, Eds., pp. 99-103, Springer Vienna, Vienna, 2011.

[10] J. J. P. Perry, D. S. Shin, E. D. Getzoff, and J. A. Tainer, "The structural biochemistry of the superoxide dismutases," Biochimica et Biophysica Acta (BBA) - Proteins and Proteomics, vol. 1804, no. 2, pp. 245-262, 2010.

[11] M. Moro, A. Almeida, J. Bolanos, and I. Lizasoain, "Mitochondrial respiratory chain and free radical generation in stroke," Free Radical Biology and Medicine, vol. 39, no. 10, pp. 12911304, 2005.

[12] J. E. Springer, P. Prajapati, and P. G. Sullivan, "Targeting the mitochondrial permeability transition pore in traumatic central nervous system injury," Neural Regeneration Research, vol. 13, no. 8, pp. 1338-1341, 2018.

[13] S. Hurst, J. Hoek, and S.-S. Sheu, "Mitochondrial $\mathrm{Ca}^{2+}$ and regulation of the permeability transition pore," Journal of Bioenergetics and Biomembranes, vol. 49, no. 1, pp. 27-47, 2017.

[14] F. Marzatico, P. Gaetani, V. Silvani, D. Lombardi, E. Sinforiani, and R. R. Baena, "Experimental isobaric subarachnoid hemorrhage: regional mitochondrial function during the acute and late phase," Surgical Neurology, vol. 34, no. 5, pp. 294-300, 1990.

[15] P. Wu, Y. Li, S. Zhu et al., "Mdivi-1 alleviates early brain injury after experimental subarachnoid hemorrhage in rats, possibly via inhibition of Drp1-activated mitochondrial fission and oxidative stress," Neurochemical Research, vol. 42, no. 5, pp. 1449-1458, 2017.

[16] T. Zhang, P. Wu, J. H. Zhang et al., "Docosahexaenoic acid alleviates oxidative stress-based apoptosis via improving mitochondrial dynamics in early brain injury after subarachnoid hemorrhage," Cellular and Molecular Neurobiology, vol. 38, no. 7, pp. 1413-1423, 2018.

[17] Y. Cui, X. Duan, H. Li et al., "Hydrogen sulfide ameliorates early brain injury following subarachnoid hemorrhage in rats," Molecular Neurobiology, vol. 53, no. 6, pp. 3646-3657, 2016.

[18] Q. Li, Y. Chen, B. Li et al., "Hemoglobin induced NO/CGMP suppression deteriorate microcirculation via pericyte phenotype transformation after subarachnoid hemorrhage in rats," Scientific Reports, vol. 6, no. 1, 2016.

[19] V. P. Nakka, P. Prakash-babu, and R. Vemuganti, "Crosstalk between endoplasmic reticulum stress, oxidative stress, and autophagy: potential therapeutic targets for acute CNS injuries," Molecular Neurobiology, vol. 53, no. 1, pp. 532-544, 2016.

[20] J. D. Malhotra and R. J. Kaufman, "Endoplasmic reticulum stress and oxidative stress: a vicious cycle or a double-edged sword?," Antioxidants \& Redox Signaling, vol. 9, no. 12, pp. 2277-2294, 2007.

[21] Y.-K. Xie, X. Zhou, H.-T. Yuan et al., "Resveratrol reduces brain injury after subarachnoid hemorrhage by inhibiting oxidative stress and endoplasmic reticulum stress," Neural Regeneration Research, vol. 14, no. 10, pp. 1734-1742, 2019.

[22] W. Xu, L. Gao, T. Li, J. Zheng, A. Shao, and J. Zhang, "Apelin13 alleviates early brain injury after subarachnoid hemorrhage via suppression of endoplasmic reticulum stress-mediated apoptosis and blood-brain barrier disruption: possible involvement of ATF6/CHOP pathway," Neuroscience, vol. 388, pp. 284-296, 2018.

[23] W. Xu, T. Li, L. Gao et al., "Apelin-13/APJ system attenuates early brain injury via suppression of endoplasmic reticulum stress-associated TXNIP/NLRP3 inflammasome activation and oxidative stress in a AMPK-dependent manner after 
subarachnoid hemorrhage in rats," Journal of Neuroinflammation, vol. 16, no. 1, 2019.

[24] W. Xu, J. Mo, U. Ocak et al., "Activation of Melanocortin 1 receptor attenuates early brain injury in a rat model of subarachnoid hemorrhage viathe suppression of neuroinflammation through AMPK/TBK1/NF-KB pathway in rats," Neurotherapeutics, vol. 17, no. 1, pp. 294-308, 2020.

[25] K. Zhang and R. J. Kaufman, "From endoplasmic-reticulum stress to the inflammatory response," Nature, vol. 454, no. 7203, pp. 455-462, 2008.

[26] J.-Y. Lee, R. F. Keep, Y. He, O. Sagher, Y. Hua, and G. Xi, "Hemoglobin and Iron handling in brain after subarachnoid hemorrhage and the effect of deferoxamine on early brain injury," Journal of Cerebral Blood Flow \& Metabolism, vol. 30, no. 11, pp. 1793-1803, 2010.

[27] P. G. Matz, M. Fujimura, A. Lewen, Y. Morita-Fujimura, and P. H. Chan, "Increased cytochrome c-mediated DNA fragmentation and cell death in manganese-superoxide dismutase-deficient mice after exposure to subarachnoid hemolysate," Stroke, vol. 32, no. 2, pp. 506-515, 2001.

[28] S.-J. Won, D.-Y. Kim, and B.-J. Gwag, "Cellular and molecular pathways of ischemic neuronal death," BMB Reports, vol. 35, no. 1, pp. 67-86, 2002.

[29] K. Osuka, Y. Suzuki, Y. Watanabe, M. Takayasu, and J. Yoshida, "Inducible cyclooxygenase expression in canine basilar artery after experimental subarachnoid hemorrhage," Stroke, vol. 29, no. 6, pp. 1219-1222, 1998.

[30] K. Osuka, Y. Watanabe, K. Yamauchi et al., "Activation of the JAK-STAT signaling pathway in the rat basilar artery after subarachnoid hemorrhage," Brain Research, vol. 1072, no. 1, pp. 1-7, 2006.

[31] P. Vigne and C. Frelin, "Endothelins activate phospholipase A2 in brain capillary endothelial cells," Brain Research, vol. 651, no. 1-2, pp. 342-344, 1994.

[32] Z.-N. Ye, L.-Y. Wu, J.-P. Liu et al., "Inhibition of leukotriene B4 synthesis protects against early brain injury possibly via reducing the neutrophil-generated inflammatory response and oxidative stress after subarachnoid hemorrhage in rats," Behavioural Brain Research, vol. 339, pp. 19-27, 2018.

[33] M. Peters-Golden and W. R. Henderson, "Leukotrienes," New England Journal of Medicine, vol. 357, no. 18, pp. 1841-1854, 2007.

[34] M. Di Napoli and I. M. Shah, "Neuroinflammation and cerebrovascular disease in old age: a translational medicine perspective," Journal of Aging Research, vol. 2011, Article ID 857484, 18 pages, 2011.

[35] P. S. Garry, M. Ezra, M. J. Rowland, J. Westbrook, and K. T. S. Pattinson, "The role of the nitric oxide pathway in brain injury and its treatment - from bench to bedside," Experimental Neurology, vol. 263, pp. 235-243, 2015.

[36] C. Iadecola, "Bright and dark sides of nitric oxide in ischemic brain injury," Trends in Neurosciences, vol. 20, no. 3, pp. 132-139, 1997.

[37] A. Woszczyk, W. Deinsberger, and D. K. Biker, "Nitric oxide metabolites in cisternal CSF correlate with cerebral vasospasm in patients with a subarachnoid haemorrhage," Acta Neurochirurgica, vol. 145, no. 4, pp. 257-264, 2003.

[38] F. A. Sehba, A. Y. Schwartz, I. Chereshnev, and J. B. Bederson, "Acute decrease in cerebral nitric oxide levels after subarachnoid hemorrhage," Journal of Cerebral Blood Flow \& Metabolism, vol. 20, no. 3, pp. 604-611, 2000.
[39] K. W. Park, C. Metais, H. B. Dai, M. E. Comunale, and F. W. Sellke, "Microvascular endothelial dysfunction and its mechanism in a rat model of subarachnoid hemorrhage," Anesthesia and Analgesia, vol. 92, no. 4, pp. 990-996, 2001.

[40] B.-L. Sun, C.-B. Zheng, M.-F. Yang, H. Yuan, S.-M. Zhang, and L.-X. Wang, "Dynamic alterations of cerebral pial microcirculation during experimental subarachnoid hemorrhage," Cellular and Molecular Neurobiology, vol. 29, no. 2, pp. 235-241, 2009.

[41] S. Yamamoto, S. Nishizawa, T. Yokoyama, H. Ryu, and K. Uemura, "Subarachnoid hemorrhage impairs cerebral blood flow response to nitric oxide but not to cyclic GMP in large cerebral arteries," Brain Research, vol. 757, no. 1, pp. 19, 1997.

[42] Y. Gilgun-Sherki, "Antioxidant therapy in acute central nervous system injury: current state," Pharmacological Reviews, vol. 54, no. 2, pp. 271-284, 2002.

[43] S. A. Lipton and J. S. Stamler, "Actions of redox-related congeners of nitric oxide at the NMDA receptor," Neuropharmacology, vol. 33, no. 11, pp. 1229-1233, 1994.

[44] F. Serrano, N. S. Kolluri, F. B. Wientjes, J. P. Card, and E. Klann, "NADPH oxidase immunoreactivity in the mouse brain," Brain Research, vol. 988, no. 1-2, pp. 193-198, 2003.

[45] S. P. Tammariello, M. T. Quinn, and S. Estus, "T NADPH oxidase contributes directly to oxidative stress and apoptosis in nerve growth factor-deprived sympathetic neurons," The Journal of Neuroscience, vol. 20, no. 1, 2000.

[46] R. P. Ostrowski, J. Tang, and J. H. Zhang, "Hyperbaric oxygen suppresses NADPH oxidase in a rat subarachnoid hemorrhage model," Stroke, vol. 37, no. 5, pp. 1314-1318, 2006.

[47] T. Nishino, K. Okamoto, B. T. Eger, E. F. Pai, and T. Nishino, "Mammalian xanthine oxidoreductase - mechanism of transition from xanthine dehydrogenase to xanthine oxidase," FEBS Journal, vol. 275, no. 13, pp. 3278-3289, 2008.

[48] F. Facchinetti, V. L. Dawson, and T. M. Dawson, "Free radicals as mediators of neuronal injury," Cellular and molecular neurobiology, vol. 18, no. 6, pp. 667-682, 1998.

[49] T. Fumoto, M. Naraoka, T. Katagai, Y. Li, N. Shimamura, and H. Ohkuma, "The role of oxidative stress in microvascular disturbances after experimental subarachnoid hemorrhage," Translational Stroke Research, vol. 10, no. 6, pp. 684-694, 2019.

[50] Y. Han, J. Su, X. Liu, Y. Zhao, C. Wang, and X. Li, "Naringin alleviates early brain injury after experimental subarachnoid hemorrhage by reducing oxidative stress and inhibiting apoptosis," Brain Research Bulletin, vol. 133, pp. 42-50, 2017.

[51] D. Zhang, H. Yan, H. Li et al., “TGF $\beta$-activated kinase 1 (TAK1) inhibition by 5Z-7-oxozeaenol attenuates early brain injury after experimental subarachnoid hemorrhage," Journal of Biological Chemistry, vol. 290, no. 32, pp. 19900-19909, 2015.

[52] Z.-y. Zhang, M. Jiang, J. Fang et al., "Enhanced therapeutic potential of nano-curcumin against subarachnoid hemorrhage-induced blood-brain barrier disruption through inhibition of inflammatory response and oxidative stress," Molecular Neurobiology, vol. 54, no. 1, pp. 1-14, 2017.

[53] H. Lv, Q. Liu, J. Zhou, G. Tan, X. Deng, and X. Ci, “Daphnetinmediated Nrf2 antioxidant signaling pathways ameliorate tertbutyl hydroperoxide (t-BHP)-induced mitochondrial dysfunction and cell death," Free Radical Biology and Medicine, vol. 106 , pp. $38-52,2017$. 
[54] S. Magesh, Y. Chen, and L. Hu, "Small molecule modulators of Keap1-Nrf2-ARE pathway as potential preventive and therapeutic Agents," Medicinal Research Reviews, vol. 32, no. 4, pp. 687-726, 2012.

[55] P. J. Syapin, "Regulation of haeme oxygenase-1 for treatment of neuroinflammation and brain disorders: regulating HO-1 in the CNS," British Journal of Pharmacology, vol. 155, no. 5, pp. 623-640, 2008.

[56] Z.-y. Zhang, M.-f. Yang, T. Wang et al., "Cysteamine alleviates early brain injury via reducing oxidative stress and apoptosis in a rat experimental subarachnoid hemorrhage model," Cellular and Molecular Neurobiology, vol. 35, no. 4, pp. 543-553, 2015.

[57] G. Kusaka, M. Ishikawa, A. Nanda, D. N. Granger, and J. H. Zhang, "Signaling pathways for early brain injury after subarachnoid hemorrhage," Journal of Cerebral Blood Flow \& Metabolism, vol. 24, no. 8, pp. 916-925, 2004.

[58] M. Cao, J. Jiang, Y. I. F. E. N. G. Du, and P. Yan, “Mitochondria-targeted antioxidant attenuates high glucose-induced P38 MAPK pathway activation in human neuroblastoma cells," Molecular Medicine Reports, vol. 5, no. 4, pp. 929-934, 2012.

[59] Y. Han, T. Zhang, J. Su et al., "Apigenin attenuates oxidative stress and neuronal apoptosis in early brain injury following subarachnoid hemorrhage," Journal of Clinical Neuroscience, vol. 40, pp. 157-162, 2017.

[60] Y. Dou, H. Shen, D. Feng et al., "Tumor necrosis factor receptor-associated factor 6 participates in early brain injury after subarachnoid hemorrhage in rats through inhibiting autophagy and promoting oxidative stress," Journal of Neurochemistry, vol. 142, no. 3, pp. 478-492, 2017.

[61] G. S. Hotamisligil and R. J. Davis, "Cell signaling and stress responses," Cold Spring Harbor Perspectives in Biology, vol. 8, no. 10, article a006072, 2016.

[62] Y. Luo, Y. Fang, R. Kang et al., "Inhibition of EZH2 (enhancer of zeste homolog 2) attenuates neuroinflammation via H3k27me3/SOCS3/TRAF6/NF-KB (trimethylation of histone 3 lysine 27/suppressor of cytokine signaling 3/tumor necrosis factor receptor family $6 /$ nuclear factor- $K \mathrm{~B}$ ) in a rat model of subarachnoid hemorrhage," Stroke, vol. 51, no. 11, pp. 33203331, 2020.

[63] S. Mitchell, J. Vargas, and A. Hoffmann, "Signaling via the $\mathrm{NF} \kappa \mathrm{B}$ system: signaling via the NF $\kappa \mathrm{Bs}$," Wiley Interdisciplinary Reviews: Systems Biology and Medicine, vol. 8, no. 3, pp. 227241, 2016.

[64] Z. Liu, D. He, X. Zhang et al., "Neuroprotective effect of early and short-time applying sophoridine in PMCAO rat brain: down-regulated TRAF6 and up-regulated p-ERK1/2 expression, ameliorated brain infaction and edema," Brain Research Bulletin, vol. 88, no. 4, pp. 379-384, 2012.

[65] J. Mo, B. Enkhjargal, Z. D. Travis et al., "AVE 0991 attenuates oxidative stress and neuronal apoptosis via mas/PKA/CREB/UCP-2 pathway after subarachnoid hemorrhage in rats," Redox Biology, vol. 20, pp. 75-86, 2019.

[66] S. Figueroa, M. J. Oset-Gasque, C. Arce, C. J. Martinez-Honduvilla, and M. P. González, "Mitochondrial involvement in nitric oxide-induced cellular death in cortical neurons in culture," Journal of Neuroscience Research, vol. 83, no. 3, pp. 441-449, 2006.

[67] M. Tanaka, M. Naoi, Y. Kato, W. Maruyama, and M. Shamoto-Nagai, "Oxidative stress in mitochondria: the involvement in neurodegenerative diseases," in Oxidative
Stress, Inflammation, and Health, Y.-J. Surh and L. Packer, Eds., vol. 20055481, pp. 423-444, CRC Press, Oxidative Stress and, 2005.

[68] T. Wang, L. Xu, L. Gao et al., "Paeoniflorin attenuates early brain injury through reducing oxidative stress and neuronal apoptosis after subarachnoid hemorrhage in rats," Metabolic Brain Disease, vol. 35, no. 6, pp. 959-970, 2020.

[69] S. P. Cregan, J. G. MacLaurin, C. G. Craig et al., "Bax-dependent caspase-3 activation is a key determinant in P53induced apoptosis in neurons," The Journal of Neuroscience, vol. 19, no. 18, pp. 7860-7869, 1999.

[70] M. Méndez-Armenta, C. Nava-Ruíz, D. Juárez-Rebollar, E. Rodríguez-Martínez, and P. Yescas Gómez, "Oxidative stress associated with neuronal apoptosis in experimental models of epilepsy," Oxidative Medicine and Cellular Longevity, vol. 2014, Article ID 293689, 12 pages, 2014.

[71] Y. Gürsoy-Özdemir, A. Can, and T. Dalkara, "Reperfusioninduced oxidative/nitrative injury to neurovascular unit after focal cerebral ischemia," Stroke, vol. 35, no. 6, pp. 14491453, 2004.

[72] Y. Handa, M. Kaneko, H. Takeuchi, A. Tsuchida, H. Kobayashi, and T. Kubota, "Effect of an antioxidant, Ebselen, on development of chronic cerebral vasospasm after subarachnoid hemorrhage in primates," Surgical Neurology, vol. 53, no. 4, pp. 323-329, 2000.

[73] M. M. Berger, L. Soguel, A. Shenkin et al., "Influence of early antioxidant supplements on clinical evolution and organ function in critically ill cardiac surgery, major trauma, and subarachnoid hemorrhage patients," Critical Care, vol. 12, no. 4, 2008.

[74] E. D. Hall, J. A. Wang, D. M. Miller, J. E. Cebak, and R. L. Hill, "Newer pharmacological approaches for antioxidant neuroprotection in traumatic brain injury," Neuropharmacology, vol. 145, no. Part B, pp. 247-258, 2019. 